\title{
Speed Image Encryption Scheme using Dynamic Galois Field GF(P) Matrices
}

\author{
Hala Bahjat, PhD \\ University of Technology \\ Computer Science Department
}

\author{
May A. Salih \\ Babylon University \\ Physical education collage
}

\begin{abstract}
Encryption security and encryption speed are two important aspects of image encryption algorithms. Due to their increasingly large size, video images present a great challenge to currently available cryptographic algorithms; the processes of encryption and decryption of images are so computationally intensive that they introduce delays beyond acceptable real-time application limits. [1] In this paper we introduce a new algorithm that uses dynamic square matrices as both encryption keys and the control stream to verify which key will be used for each block.

The study case showed in this paper works on $\operatorname{GF}(7)$ and for encryption key sizes varying from $3 \mathrm{X} 3$ to $12 \mathrm{X} 12$ The goal is to provide a highly secure encryption algorithm with a wide space for encryption speed.
\end{abstract}

\section{General Terms}

Pattern Recognition, Security, Algorithms ,Galois fields

\section{Keywords}

Galois field, real time, image encryption

\section{INTRODUCTION}

We ask that authors follow some simple guidelines. In essence, we ask you to make your paper look exactly like this document. The easiest way to do this is simply to download the template, and replace the content with your own material.

During the development of network and multimedia technology, more and more images transmit over the Internet and through the wireless networks. Digital images have become one of the most important information carriers, which is helpful for people to communicate with each other. However, because of the intrinsic features of images, such as bulk data capacity and high correlation among pixels, it is not suitable for practical image encryption, especially during online communications. Therefore, people begin to explore dynamic Galois field matrices which is more efficient at hiding image information. [2]

Image encryption has applications in internet communication, multimedia systems, medical imaging, telemedicine, military communication, etc. Images differ from text in that they are bigger in size and the decrypted image must be equal to the encrypted image. Another significant point to consider in transfer of digital images is their special attributes like bulk data capacity, high redundancy, and high correlation between neighboring pixels [3][4].

\section{IMAGE CRYPTOGRAPHY}

Cryptography presents the best, and an inevitable, solution to problems in sending images. Cryptography can be defined as the art of creating an unintelligible form from intelligent information [6]. It is done to ensure that if any eavesdrop attempts to deduce the plaintext, then he is unable to discover this confidential data. It is an ancient technology for the secure transfer of messages. Traditional use of cryptography dealt only with textual data and not much focus was laid on the secure transmission of images or audio/visual data. But today, the situation has changed and now it is capable for handling any type of media or data. Encryption of textual data is relevant mostly to one-dimensional data, which is why techniques best suited for text are inadequate for twodimensional digital images. Moreover, cryptography has spread gradually from its early use in military and political fields into everyday civilian life. It now plays a crucial role in activities like Internet banking, e-commerce, e-finance, etc.[7].

\section{TRADITIONAL ENCRYPTION STANDARDS}

The established encryption algorithms like DES, AES, etc concentrate on changing two-dimensional data into onedimensional data and then applying encryption on it. [3] But this technique is less efficient to encrypt and decrypt images. Because the most important factor in the encryption process is time of this reason, such a primitive style of encryption it is not preferred for digital images. Recently, Zhang et al. proposed an image encryption method based on a total shuffling scheme [11]. In this method, the secret encryption code stream is not only associated with the key, but also related to the plain image. Because the random number used in the diffusion process is obtained by iterating the skew tent map, and the number of iterations is determined by the previous pixel value of cipher image, which includes the plain image's previous pixel value, the next random number is indirectly related to the previous pixel value of plain image. This plain image encryption method holds up strongly against plaintext attacks [12]. However, the first secret code in [11] is not safe enough to resist the chosen plaintext attack.

\section{GAUSS JORDAN ELIMINATION METHOD}

Using the usual notation, by $\mathbb{C}_{r}^{m \times n}$ we denote the set of all complex $m \times n$ matrices of $\operatorname{rank} r$, and by $I$ we denote the unit matrix of an appropriate order. Furthermore $\mathrm{A}^{*}, \mathcal{R}(A)$, rank (A) and $\mathcal{N}(A)$ denote the conjugate transpose, the range, the rank, and null space of $A \in \mathbb{C}^{m \times n}$. If $A \in \mathbb{C}_{r}^{m \times n}$. $T$ is a subspace of $\mathbb{C}^{n}$ of dimension $t \leq r$ and $S$ is subspace of $\mathbb{C}^{m}$ of dimension $m$ - $t$, then $\mathrm{A}$ has a $\{2\}$ - inverse $\mathrm{X}$ such that $\mathcal{R}(X)=$ $T$ and $\mathcal{N}(X)=S \quad$ if and only if $A T \oplus S=\mathbb{C}^{m}$. In the case where its existence is ensured, $X$ is unique and denoted by $A_{T S S}^{(2)}$ [8]. We study Gauss Jordan elimination methods for computing various inverses of square matrices. The oldest and best known among these methods is for calculating the inverse matrix. The Gauss Jordan elimination method for computing the inverse of a nonsingular matrix $\mathrm{A}$ is based on the executing elementary row operations on the pair $[\mathrm{A} \mid \mathrm{I}]$ 
and its transformation into the 7block matrix involving the inverse $\mathrm{A}^{-1}$. A number of numerical methods are developed for computing various classes of outer inverses with the prescribed range and null space. The Gauss Jordan elimination method to compute the Moore Penrose inverse is developed in [9]. The method from [9] is based on two successive sets of elementary row operations.

\section{THE PROPOSED IMAGE ENCRYPTION ALGORITHM}

The proposed schema is based on two types of keys: encryption key and control key. The control key is generated using a controlled ranged randomized stream and the encryption keys are a set of non-fixed variable $(\mathrm{GF}(\mathrm{P}))$ matrices

The first step converts the matrix from 2D to a stream of data. The next step multiplies variable-sized blocks with the opponent key matrix based on the control key as showed in fig (1).

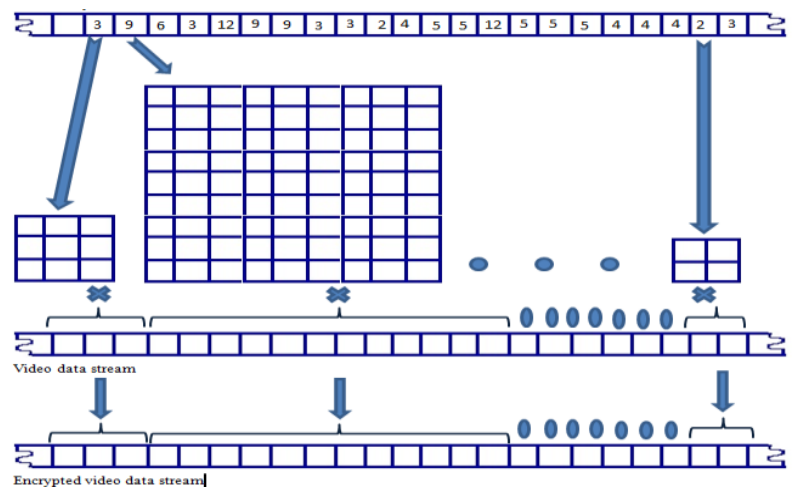

Fig (1) Proposed Encryption Scheme

Encryption in digital images mostly works at pixel level, which is the lowest level of information in the image. But because of strong correlation between neighboring pixels, one can easily decode data for one pixel if that of a neighboring pixel becomes known. However, an image can also be interpreted as an ordered arrangement of image blocks instead of pixels. An accurate orientation of these image blocks lets us infer information from the image, where any change causes visual disruption [4]. The proposed algorithm is shown in fig (2). Thus, using block level encryption for images will help overcome the chief nuisance in image encryption, correlation between neighboring pixels. The block size should be smaller for better transformation because then fewer pixels will keep their neighbor's data [6]. These advantages of blocks over pixels are why block encryption is preferred over stream cipher. However, a considerable drawback of block cipher is that it produces the same cipher text for the same plaintext if it is encoded with the same key. In response, we propose an image encryption technique of a partial symmetric-key algorithm.it is not fully dependent on the secret key and hence achieves better computational security against unauthorized attacks. It actually uses two keys for encoding; one at the block level and other at the pixel level.

Step 1. Generate Control Key

Step 2. Generate GF(p) masks and inverses for sizes [3-12] using Gauss Jordan elimination Method

Step 3. Reshape image data file from 2-D to 1-D

Step 4. Repeat until end of file
1. Pick control key value

2. Select mask dimension $=$ control key value

3. Determine size of block $=$ control key value

4. Apply function like encrypted block $=$ mask $*$ block

5. Control key controller +1

6. Check or end of file

Step 5. Collect encrypted blocks to form encrypted image Step 6. Reshape 1-D data to 2-D image data file

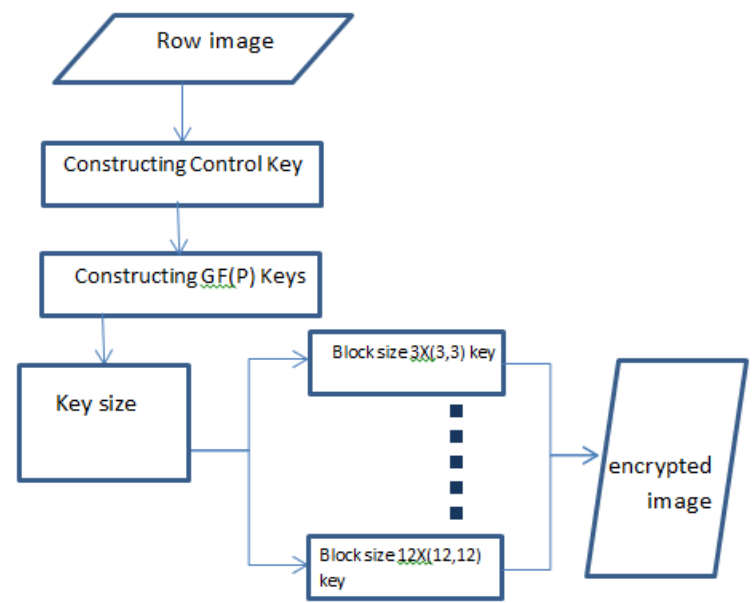

Fig(2) Proposed Algorithm Flow chart

\section{PERFORMANCE OF THE PROPOSED ALGORITHM}

A good image encryption algorithm should be sensitive to the cipher keys, and the key space should be large enough to make brute force attacks infeasible. For the proposed image encryption algorithm, key space analysis and testing have been performed and completed, with results summarized as follows:

Key Space is $1.8388 \mathrm{e}+118$.The proposed image cipher has (1.8388 e+118) different combinations of possible keys An image cipher with such a large combined key space is sufficient for reliable practical use and can resist all kinds of brute force attacks.

\section{EXPERIMENTAL RESULTS} Table (1) Behavior Analysis

\begin{tabular}{|l|l|l|l|}
\hline $\begin{array}{l}\text { Image size } \\
\text { (pixels) }\end{array}$ & $\begin{array}{l}\text { Average } \\
\text { encryption } \\
\text { time }\end{array}$ & $\begin{array}{l}\text { Average } \\
\text { decryption } \\
\text { time }\end{array}$ & $\begin{array}{l}\text { Average } \\
\text { total time }\end{array}$ \\
\hline $480 * 480$ & $0.04 \mathrm{sec}$. & $2.5 \mathrm{sec}$. & $2.54 \mathrm{sec}$. \\
\hline $720 * 720$ & $2.7 \mathrm{sec}$. & $3.1 \mathrm{sec}$. & $5.8 \mathrm{sec}$. \\
\hline $960 * 960$ & $8 \mathrm{sec}$. & $13.3 \mathrm{sec}$. & $21.3 \mathrm{sec}$. \\
\hline
\end{tabular}




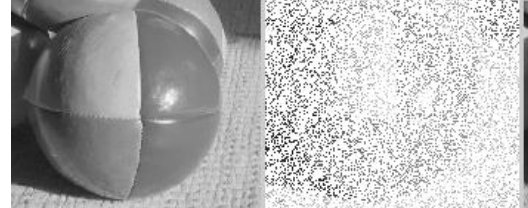

Input image (ball) encrypted image

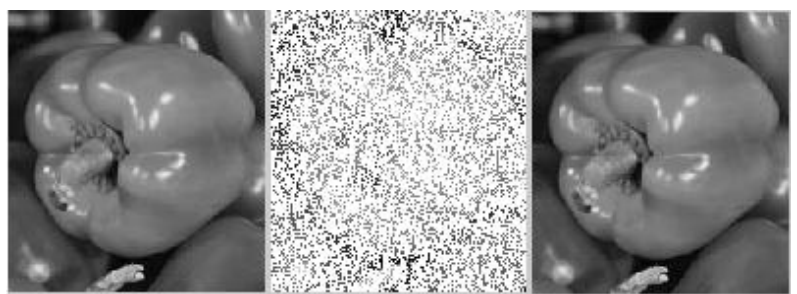

Input image (pepper) encrypted image decrypted image

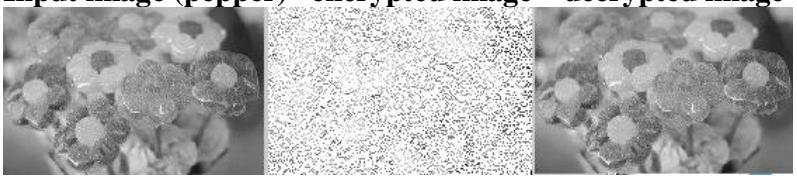

Input image (roses) encrypted image

decrypted image

Table(2) encryption / decryption time

\begin{tabular}{|c|c|c|}
\hline Image & Encryption time & Decryption time \\
\hline Ball & $0.003422 \mathrm{sec}$. & $4.239834 \mathrm{sec}$ \\
\hline Pepper & $0.002073 \mathrm{sec}$ & $0.194880 \mathrm{sec}$ \\
\hline Roses & $0.005908 \mathrm{sec}$ & $1.982690 \mathrm{sec}$ \\
\hline
\end{tabular}

\section{OBJECTIVE FIDELITY CRITERIA}

Table (3)SNR, PNSR and MSE results

\begin{tabular}{|l|l|l|l|}
\hline image & NSR & PNSR & MSE \\
\hline Ball & 3.0634 & 6.7695 & $1.3255 \mathrm{e}+04$ \\
\hline Pepper & 4.0483 & 5.9599 & $1.6485 \mathrm{e}+04$ \\
\hline Roses & 3.4704 & 7.6080 & $1.1015 \mathrm{e}+04$ \\
\hline
\end{tabular}

The objective fidelity criteria provide equations that can measure the amount of error in the reconstructed (deciphered) images or to measure the amount of error between pure image and ciphered image. Commonly used objective measures are the Root-Mean-Square- error (RMSE), Signal-to-Noise Ratio (SNR) and the Peak Signal-to-Noise Ratio (PSNR) [Sco98] .

\section{1 (The Root- Mean- Square Error (RMSE))}

The MSE is the average of the square of errors (pixel differences) of the two images, found by taking the square root ("root") of the error squared ("square") divided by the total number of pixels in the image ("mean") [Ikh05]:

$$
M S E=\frac{1}{H * W} \sum_{y=0}^{H-1 W-1} \sum_{x=0}\left(f(x, y)-f^{\prime}(x, y)^{2}\right)
$$

The Root Mean Square error (RMSE) is defined as the square root of the MSE:

$$
R M S E=\left[\frac{1}{H * W} \sum_{y=0}^{H-1 W-1} \sum_{x=0}\left(f(x, y)-f^{\prime}(x, y)\right)^{2}\right]^{1 / 2}
$$

Hence, the smaller the value of MSE, the better the deciphered image represents the original image and the large value of error. The better the ciphered image, the more pure image information it conceals.

\section{2 (Signal to Noise Ratio)}

It is fidelity parameter used to measure the distortion level caused by image cryptography. It can be defined as:

$$
S N R=\frac{\sum_{y=0}^{H-1} \sum_{x=0}^{W-1}(f(x, y))^{2}}{\sum_{y=0}^{H-1 W-1} \sum_{x=0}\left(f(x, y)-f^{\prime}(x, y)\right)^{2}}
$$

\subsection{Peak Signal to Noise Ratio (PSNR)}

This can be defined as:

$$
P S N R=10 \log _{10}\left(\frac{(255)^{2}}{M S E}\right)
$$

Where MSE is the mean square error.

In image cryptography, a large PSNR value implies a betterdeciphered image, and a smaller number implies better image concealment of original image is obtained. Subsequent Pages

For pages other than the first page, start at the top of the page, and continue in double-column format. The two columns on the last page should be as close to equal length as possible.

\section{CONCLUSION \\ 9.1 Trade-off between complexity and security}

For multimedia content encryption, especially for application in real-time video communication, low processing overhead becomes extremely important. Due to the restriction of realtime, it becomes important to select the most critical unit to shuffle. Syntax compliance makes it easier to locate different types of critical data blocks in the process of bit stream analysis.

\subsection{Security Analysis}

Due to the good randomicity of pseudo-random sequence, the encryption approach presented in this paper amounts to changing the cipher every time. According to the theory of cryptology, it is the most secure encryption approach and is therefore superior to methods using a standard encryption algorithm.

In this paper, we proposed a lookup table method to improve the encryption/decryption speed of the image encryption scheme presented in $[10,11]$, and employed "addition and modulo" operator instead of "XOR" in the diffusion procedure. We compared our proposal and the methods in [10, 11] regarding encryption/decryption speed, key space, key sensitivity, information entropy, cipher image statistical properties, and plain image sensitivity analysis. The results fully demonstrate that the speed of our proposed method is about 8 times faster than those of $[10,11]$ and with stronger, more complex security. Therefore, the proposed method is more feasible for practical communications. 


\section{REFERENCES}

[1] "Performance Study of a Selective Encryption Scheme for the Security of Networked, Real-Time Video "Las Vegas, Nevada, USA September 20-September 23 ISBN: 0-8186-7180-7

[2] "A Symmetric Image Encryption Scheme Based on Composite Chaotic Dispersed Dynamics System" Zhenzhen Lv1, Lei Zhang2, and Jiansheng Guo 3,Proceedings of the Second Symposium International Computer Science and Computational Technology(ISCSCT '09) Huangshan, P. R. China, 2628,Dec. 2009, pp. 191-194

[3] Zhang S. and M. A. Karim, pp. 318-322, Vol. 21, No. 5, June 5 1999, Color image encryption using double random phase encoding, Microwave and optical technology letters.

[4] Maniccam S.S and Bourbakis N.G., pp. 1229-1245, 2001, Lossless image compression and encryption using SCAN, Pattern Recognition.

[5] Vinod Patidar G. Purohit, K. K. Sud, N. K. Pareek, "Image encryption through a novel permutation substitution scheme based on chaotic standard map," Chaos-Fractal Theory and its Applications, IEEE International Workshop on, 2010, pp. 164-169.

[6] Quist-Aphetsi Kester, "A cryptographic Image Encryption technique based on the RGB Pixel shuffling," International Journal of Advanced Research in Computer
Engineering \& Technology (IJARCET), vol. 2, issue 2, January 2013, pp. 848-854

[7] The Hacking Tutorial Website. [Online]. Available: http://www.hacking-tutorial.com/tips-andtrick/hidesecret-file-inside-an-image-steganography/

[8] "Sparse Matrix Technology" 2007 by Sergio Pissanetzky and SciControls.com. ISBN 978-0-9762775-3-8 .

[9] "Gauss Jordan elimination method for computing outer inverses " ,Predrag S. Stanimirovi_c1, Marko D. Petkovi_c2.

[10] A. A. Adb El-Latif, L. Li, T. Zhang, N. Wang, X. Song, and X. Niu, "Digital image encryption scheme based on multiple chaotic systems," Sensing. Imaging. An Int. J. vol. 13, pp. 67-88, 2012.

[11] G. Zhang, and Q. Liu, "A novel image encryption method based on total shuffling scheme," Opt. Commun. vol. 284, pp. 2775-2780, 2011.

[12] Y. Zhang, J. Xia, P. Cai, and B. Chen, "Plaintext related two-level secret key image encryption scheme," TELKOMNIKA. vol. 10, pp. 1254-1262, 2012.

[13] Yong Zhang "Encryption Speed Improvement on "An Improvement over An Image Encryption Method Based on Total Shuffling" 2013 International Conference on Sensor Network Security Technology and Privacy Communication System (SNS \& PCS) 